\title{
A relevância da atividade física e exercício físico em tempos pandêmicos: Um olhar para
} a saúde e qualidade de vida

The relevance of physical activity and physical exercise in pandemic times: A look at health and quality of life

La relevancia de la actividad física y el ejercicio físico en tiempos de pandemia: Una mirada a la salud y la calidad de vida

Resumo

Diante do momento pandêmico instaurado pelo coronavírus (SARS-CoV-2) causador da doença infecciosa denominada COVID-19, despertou-se a necessidade de discutir alternativas de diminuir os impactos na saúde e qualidade de vida da população, tendo o exercício e a atividade física como ferramenta crucial para a manutenção, potencialização e promoção da saúde no contexto em tempos de pandemia. Com isso, o presente trabalho de pesquisa apresenta os impactos associados a Atividade e ao Exercício Físico em tempos difíceis, como o período de isolamento social, trazendo uma visão holística para a saúde e qualidade de vida da sociedade. Assim, esta revisão bibliográfica tem o objetivo de apresentar e analisar a relevância do Exercício Físico e Atividade Física em meio à pandemia da COVID-19, abordando cuidados e benefícios para o ser humano, atrelando a saúde e a qualidade de vida do indivíduo no contexto social. Neste estudo discutiremos também relação de aplicabilidade e a importância da orientação de um profissional de Educação Física respeitando as linhas de orientações para a melhor e mais segura aplicação de Exercícios Físicos para cada público. A metodologia utilizada foi a pesquisa bibliográfica, com abordagem descritiva, baseada em livros e artigos já publicados, encontrados na base de dados dos sites PubMed, Portal Periódico Capes, Google acadêmico e Scielo. Os resultados nos mostram que através da realização de exercícios físicos é possível mensurar o quanto essa prática é fundamental para a manutenção da saúde e qualidade de vida dos indivíduos na sociedade, em um momento delicado como a pandemia, que mudou a rotina de vida da população, bem como trouxe à tona a compreensão dos benefícios que as atividades físicas promovem na vida do ser humano.

Palavras-chave: Educação física; Exercício físico; Pandemia; Atividade física. 


\begin{abstract}
In view of the pandemic period brought about by the coronavirus (SARS-CoV-2) that causes the infectious disease known as COVID-19, the need arose to discuss alternatives to reduce the impacts on the population's health and quality of life, with exercise and activity as a crucial tool for the maintenance, enhancement and promotion of health in the context of a pandemic. Thus, this research work presents the impacts associated with Activity and Physical Exercise in difficult times, such as the period of social isolation, bringing a holistic view to society's health and quality of life. Thus, this literature review aims to present and analyze the relevance of Physical Exercise and Physical Activity in the midst of the COVID-19 pandemic, addressing care and benefits for human beings, linking the health and quality of life of the individual in the context Social. In this study we will also discuss the applicability relationship and the importance of the guidance of a Physical Education professional, respecting the guidelines for the best and safest application of Physical Exercises for each audience. The methodology used was bibliographic research, with a descriptive approach, based on books and articles already published, found in the database of the websites PubMed, Portal Periodical Capes, Google Academic and Scielo. The results show that through physical exercise it is possible to measure how fundamental this practice is to maintain the health and quality of life of individuals in society, in a delicate moment such as the pandemic, which changed the population's life routine, as well as brought to light the understanding of the benefits that physical activities promote in the human being's life.
\end{abstract}

Keywords: Physical education; Physical exercise; Pandemic; Physical activity.

\title{
Resumem
}

Ante el período pandémico provocado por el coronavirus (SARS-CoV-2) causante de enfermedad infecciosa conocida como COVID-19, surgió la necesidad de discutir alternativas para reducir los impactos en la salud y calidad de vida de la población, con ejercicio y la actividad como herramienta fundamental para el mantenimiento, mejora y promoción de la salud en el contexto de una pandemia. Así, este trabajo de investigación presenta los impactos asociados a la Actividad y el Ejercicio Físico en momentos difíciles, como el período de aislamiento social, aportando una mirada holística a la salud y calidad de vida de la sociedad. Así, esta revisión de la literatura tiene como objetivo presentar y analizar la relevancia del Ejercicio Físico y la Actividad Física en medio de la pandemia de COVID-19, abordando el cuidado y los beneficios para el ser humano, vinculando la salud y la calidad de vida del individuo en el contexto social. En este estudio, también discutiremos la relación de aplicabilidad y la importancia de la orientación de un profesional de Educación Física, respetando las pautas para la mejor y más segura aplicación de Ejercicios Físicos para cada público. La metodología utilizada fue la investigación bibliográfica, con enfoque descriptivo, basada en libros y artículos ya publicados, encontrados en la base de datos de los sitios web PubMed, Portal Periódico Capes, Google Academic y Scielo. Los resultados muestran que a través del ejercicio físico es posible medir cuán fundamental es esta práctica para mantener la salud y la calidad de vida de los individuos en la sociedad, en un momento delicado como la pandemia, que cambió la rutina de vida de la población, así como sacó a la luz la comprensión de los beneficios que las actividades físicas promueven en la vida del ser humano.

Palabras clave: Educación física; Ejercicio físico; Pandemia; Actividad física.

\section{Introdução}

A prática de atividade física e ou exercício físico regular e orientada é de fundamental importância para a manutenção da saúde, adquirindo qualidade de vida, potencializando a prevenção de doenças que podem surgir com a inatividade física e o sedentarismo. Para OMS (2020), o sedentário é uma pessoa que gasta poucas calorias por semana com atividades do dia a dia, ocupacionais como ir ao trabalho, realizar serviços domésticos. Assim, é evidente a pratica que movimentar-se contribui significativamente para um estilo de vida ativa e saudável, este que é fundamental para um melhor estilo de vida. Nahas (2010), evidencia que a qualidade de vida está atrelada a um conjunto de ações habituais que refletem as atitudes, os valores e as oportunidades na vida das pessoas, em que devem ser valorizados elementos concorrentes ao bem estar pessoal, como controle do estresse, a nutrição equilibrada, a atividade física regular os cuidados preventivos com a saúde e o cultivo de relacionamentos sociais. O Exercício Físico se refere a um conjunto de movimentos planejados e organizados em busca de um objetivo relacionado ao condicionamento físico e qualidade de vida aumentando o desempenho do sujeito (Carvalho et al. 2021). Diante do período pandêmico que assola a população mundial o comportamento sedentário se tornou algo rotineiro, devido ao isolamento social, assim surgiram discursões sobre a prática de atividade física, exercícios físicos, recomendações e seus benefícios. 
Dentro desse viés, a temática traz uma relação entre o Exercício Físico e a Inatividade Física, tendo um olhar especial para à saúde e a qualidade de vida. Diante desse cenário social que é fragilizado em questões de comportamentos que possibilitem condições melhores de saúde e qualidade de vida, é fundamental a orientação de um profissional de Educação Física com entendimento sobre as implicações musculares, fisiológicas e ósseas, mostrando as vantagens de treinar, os cuidados e riscos do treinamento. Vale ressaltar, que para Mattos et al. (2020), o acompanhamento de um profissional de Educação Física proporciona um direcionamento da Atividade e Exercício Físico para os indivíduos que necessitam de orientações de forma específica frente a algum agravo de saúde.

Assim, é de fundamental relevância que durante todo processo de desenvolvimento de Atividade e Exercícios Físicos seja acompanhada pelo profissional de Educação Física, desta forma, Elesbão et al., (2020), salientam que a realização de exercícios físicos e atividade física de maneira saudável, sempre respeitando os limites de cada pessoa, sendo assim, indispensável o acompanhamento de um profissional de Educação Física.

É importante frisar, que o presente trabalho tem uma grande relevância, frente ao contexto social, pois possibilita aos seus leitores a compreensão frente à importância da Atividade Física e Exercício Físico em tempos difíceis, como o período de isolamento social, trazendo uma visão para a saúde e qualidade de vida na sociedade. Sendo também, para campo cientifico uma ótima alternativa para novas discussões sobre a temática de suma valia, que é a Atividade Física e Exercício Físico em tempos pandêmico, com um olhar para saúde e qualidade de vida.

Nesse sentido, o ser humano deve ter uma vida ativa frente à prática de Exercícios Físicos em período pandêmico mesmo estando em quarentena exercícios de calistenia onde segundo Santos et al., (2020) a calistenia é uma prática esportiva que o seu desempenho está ligado ao seu próprio peso corporal, tendo como objetivo, proporcionar o melhoramento da qualidade de vida, como também aspectos como força física, resistência, equilíbrio, postura, mediante a movimentos lineares, sem necessitar de aparelhos que são encontrados na academia, desta forma, uma prática que pode ser realizada em casa, sem a necessidade de ir a academia. Desta forma Elesbão et al., (2020) frisam que em tempo de epidemia é de suma relevância a prática de Atividade Física, para amenizar os aspectos negativos referente a inatividade física, tornando assim, ainda mais relevante nesse período de distanciamento social, pois devido as restrições para diminuição da propagação do corona vírus, é importante o mínimo da prática de Atividade Física e Exercício Físico, nesse período, visando a melhoria do bem-estar, saúde mental, em consonância com ascendentes níveis de Atividade Física/Exercício Físico e na diminuição de tempo com comportamento sedentário.

Esta revisão bibliográfica tem o objetivo de apresentar e analisar a relevância do Exercício Físico e Atividade Física em meio à pandemia do COVID-19, que é uma infecção respiratória aguda causada pelo coronavírus SARS-CoV-2, potencialmente grave, de elevada transmissibilidade e de distribuição global. Abordando cuidados e benefícios para o ser humano, atrelando a saúde e a qualidade de vida do indivíduo no contexto social. Neste estudo discutiremos também relação de aplicabilidade e a importância da orientação de um profissional de Educação Física respeitando as linhas de orientações para a melhor e mais segura aplicação de Exercícios Físicos para cada público.

\section{Metodologia}

O trabalho é classificado como uma pesquisa bibliográfica, com abordagem descritiva, baseada em livros e artigos já publicados, encontrados na base de dados dos sites PubMed, Portal Periódico Capes, Google acadêmico e Scielo. Foram selecionados 52 artigos no total, onde foram utilizados 32 para construção do artigo, publicados entre os anos de 2016 a 2021. 
Diante dessa problemática, essa pesquisa possui um caráter bibliográfico, o qual mediante Gil (2010), igualmente a outros tipos de pesquisa, seu desenvolvimento se dá por fases, mas se desenvolve com a escolha de um tema a ser estudado e com a revisão bibliográfica de obras e periódicos já publicados, voltados ao assunto que será trabalhado, nesse caso, o Exercício Físico e Atividade Física frente há tempos pandêmicos no contexto social.

A abordagem qualitativa além de ser uma opção de investigar e estudar sobre determinado tema, ela justifica-se por ser a melhor e mais adequada forma de entender os fenômenos de natureza social (Richardson, 2011). Dentro deste viés, a pesquisa traz uma compreensão sobre a importância do estudo mediante aos aspectos do Exercício Físico e Atividade Física em tempo de pandemia, tendo um olhar para saúde e qualidade de vida.

Foram utilizadas as seguintes expressões em português e inglês para a busca dos estudos: (Exercício Físico, Atividade Física, pandemia, saúde e Qualidade de vida) em todas as pesquisas. Essas palavras também foram usadas em conjunto para pesquisar tópicos mais específicos sobre o tema. Após a leitura exploratória foi realizado uma leitura seletiva do material, verificando e relevância dos dados.

Esta revisão apresenta tópicos dos principais temas encontrados nos artigos selecionados relacionados a campo estudado. Temas como: Exercício Físico e Atividade Física e o segundo tópico a relevância da Atividade Física e Exercício Físico em tempos pandêmicos. A relevância deste artigo consiste na possibilidade de proporcionar aos profissionais de Educação Física um melhor entendimento sobre essa temática tão relevante no contexto social, que é o exercício físico e atividade física frente à saúde e qualidade de vida em tempos pandêmicos.

\subsection{Exercício físico e atividade física}

É importante frisar que no cenário atual, é notório que o exercício físico e a atividade Física, vêm desenvolvendo seu espaço na sociedade, em um contexto social que deixa a desejar em questões referentes a cuidados com a saúde e qualidade de vida, fica perceptível a relevância que o Exercício Físico e a Atividade Física têm para com indivíduo que a pratica, possibilitando que ser tornem pessoas ativas e com uma saúde e vida de qualidade.

É importante salientar, que nesse momento que vivemos de diversos obstáculos que envolve os cuidados com nossa saúde, o exercício físico é uma ferramenta cada vez mais ofertada e de relevância para uma boa qualidade de vida, nesse sentido, referente ao Exercício Físico, Carvalho et al., (2021) frisam que o Exercício Físico é caracterizado por um conjunto de movimento de maneira planejada e estruturada, visando um determinado objetivo, como por exemplo a melhoria da capacidade física ou do seu desempenho. Nesse viés, para corroborar sobre os aspectos do Exercício Físico, Zawadzki et al. (2019), salientam que o Exercício Físico sendo desenvolvido em média três vezes por semana, com uma intensidade de forma moderada ou intensa, pode proporcionar benefícios na aptidão física e na saúde, como também nas relações sociais e psicológicas.

Vale ressaltar, na relação sobre a intensidade na prática de Atividade Física, segundo Brasil (2021) que a intensidade está relacionada ao grau de esforço físico necessário, sendo de forma, leve, moderada e vigorosa, onde no aspecto leve, exige o mínimo de esforço físico, pois acarreta em menor aumento da respiração e batimentos cardíacos, já na moderada, tem predominância em um esforço físico maior, com respiração mais rápida que normalmente e tendo batimentos cardíacos de forma moderada e no último aspecto, que é vigorosa, exige grande esforço físico, com batimentos e respiração no ritmo maior que o normal, tendo em sua escala de percepção de esforço, leve de 1 a 4, moderada de 5 a 6 e vigorosa de 7 a 8 .

Carvalho et al., (2021) abordam que a prática de Exercício Físico proporciona o combate a diversas doenças, levando benefícios, sejam em curto, médio ou longo prazo, tais como, a diminuição da obesidade, de fatores de risco referente as doenças 
cardiovasculares e desta maneira, quanto mais ativos esses indivíduos, melhor será sua saúde. Partindo dessa permissa pesquisadores brasileiros publicaram ponto de vista chamando atenção sobre a importância da prática da atividade em casa ou ao ar livre, enfatizando, também, a importância da redução dos comportamentos sedentários durante a pandemia (Pitanga et al., 2020).

A atividade física é definida como qualquer movimento corporal, produzido pela musculatura esquelética, que resulta em gasto energético (Meneguci et al., 2016). Desta forma, para corroborar com o que explanado, Carvalho et al., (2021) salientam que a Atividade Física é caracterizada como qualquer movimento produzido pelo nosso corpo, onde possibilite contrações musculares, gerando acréscimo no gasto energético, para além dos níveis de repouso.

Corroborando com os pontos salientados pelos autores acima, Brasil (2021) traz que atividade física está ligada aos movimentos de forma voluntaria do corpo, com gasto energético maior que seu nível de repouso, onde possibilita interação social e com ambiente, sendo desenvolvido em momentos, tais como, tempo livre, estudo, trabalho, deslocamento e nas tarefas domesticas.

Assim, vale ressaltar sobre Atividade Física e Exercício Físico, segundo Florêncio Júnior et al., (2020) que a prática de forma regular do Atividade Física é de fundamental importância para manutenção do peso corporal, benefícios para o aspecto cognitivo, além disso, o Exercício Físico pode acarretar efeitos satisfatórios, em funções cognitivas, promove adaptações nas estruturas cerebrais e sinápticas, que tem relevância para a cognição. Segundo a OMS (2020) as crianças e adolescentes devem fazer pelo menos uma média de 60 minutos por dia de atividade física de moderada a vigorosa intensidade, ao longo da semana, a maior parte dessa atividade física deve ser aeróbica, sendo que, para públicos adultos sem restrições médicas, deve ser praticada atividade física de forma moderada de 150 a 300 minutos e de maneira intensa de 75 a 150 minutos. Desse modo, apresentar os benefícios de diversos exercícios físicos na infância, tanto em aulas de educação física escolar como nas escolas de modalidades esportivas nos mais diferentes ambientes parece necessário no atual cenário (Carvalho, 2021).

\subsection{A relevância da atividade física e exercício físico em tempos pandêmicos}

A Organização Mundial da Saúde - OMS (2020) declarou, em 30 de janeiro de 2020, Emergência de Saúde Pública de Importância Internacional (ESPII) por causa do surto do novo coronavírus, causador da COVID-19. A situação de ESPII é o mais alto nível de alerta da OMS, e também considerada, devido ao alcance de disseminação internacional da doença, um risco de saúde pública excepcional para o planeta. Essa decisão visou criar redes de coordenação, cooperação e solidariedade global contra a propagação do vírus. Assim, como consequência do aumento de casos de contágio e, principalmente, de mortes por COVID-19 durante a pandemia, tornou-se indispensável que as medidas de controle e segurança de infecções fossem seguidas. Desta forma, a permanência em casa foi um ponto de segurança essencial para a redução do número de casos (Cortez et al, 2020).

Pensando nisso, várias medidas de proteção devem ser tomadas. Dentre elas, uma conscientização sobre práticas de higiene apropriadas e vigorosas, que vão desde a lavagem consistente das mãos ao uso persistente de máscaras. Também cabe ressaltar o papel da vigilância sanitária na fiscalização e controle dos casos regionais de incidência do vírus. Junto a essas medidas, o distanciamento social, que tem demostrado ser a estratégia mais eficiente para reduzir a disseminação do vírus no mundo (ECDC, 2020). A atividade física é recomendada à população, tendo em vista que é considerada uma ferramenta fundamental para a melhoria da saúde e qualidade de vida do ser humano. Boa parte dos estudos sobre os impactos da atividade física durante o isolamento social parte da lógica dos seus efeitos em variáveis fisiológicas, como melhoria do sistema imunológico (Ladu et al, 2020). O exercício físico não imuniza as pessoas contra a COVID-19, mas auxilia na resposta imunológica do ser humano, ou seja, uma pessoa que tem uma vida mais ativa estará menos suscetível a doenças virais, entre outras. 
Em alguns países, como no Brasil, as academias e espaços de recreação foram fechados, na intenção de diminuir a proliferação do vírus. Escolas públicas e privadas também foram fechadas, como prevenção contra aglomerações. Os baixos níveis de atividade física podem ter efeitos negativos nos processos cognitivos dos indivíduos, adicionados ao próprio estresse do momento de isolamento social (Hall et al, 2020). Segundo Alecrim (2020), as práticas de atividades físicas e de lazer podem ajudar na diminuição de sintomas de diversas doenças, dentre elas a contaminação e agravamento da COVID-19.

Assim, para corroborar com o que foi explanado, Alberti et al. (2021), salientam que a pratica de atividade física, é de grande relevância para a prevenção de doenças crônicas não transmissíveis, e para prática nesse período de pandemia, deve-se seguir medidas de proteção para que não ocorra a propagação do COVID-19, como também, estar atento à intensidade dos exercícios, pois na utilização da máscara que é um dos materiais de proteção contra COVID-19, há uma limitação na ingestão de ar, levando assim, a uma absorção de oxigênio, e desta forma, proporcionando um desconforto fisiológico, e assim, é importante a redução do volume e intensidade das atividades físicas.

No entanto, ficar muito tempo em casa pode intensificar comportamentos que levam ao sedentarismo, acarretando ou agravando diversos problemas de saúde física ou psicológica. Portanto, a atividade física regular e a rotina de exercícios em casa ou em academias - com os devidos cuidados sanitários, passam a ser uma estratégia de vida saudável durante a pandemia do novo coronavírus (Chen et al, 2020). A partir desse ponto, vale ressaltar o entendimento do Colégio Americano de Medicina do Esporte (ACSM, o qual define atividade física como qualquer movimento corporal produzida pelos músculos esqueléticos que resulta no gasto energético maior que os níveis de repouso, bem como, define exercício físico como sendo toda atividade física planejada, estruturada e repetitiva que tem por objetivo a melhoria e a manutenção da aptidão física (ACSM 2018).

Segundo a OMS (2020), a COVID-19 possui um alto poder de transmissibilidade de humano para humano e uma pessoa infectada pode transmitir a várias outras. Apesar de cerca de $80 \%$ dos infectados terem recuperação da doença sem precisar de tratamento hospitalar, uma em cada seis pessoas infectadas fica gravemente doente e desenvolve dificuldade de respirar e muitas já são as mortes decorrentes dessas complicações (OMS, 2020). A Organização ainda alertou que as pessoas idosas e as que têm outras condições de saúde como pressão alta, problemas cardíacos e do pulmão, diabetes ou câncer, teriam maior risco de ficarem gravemente doentes, no entanto, qualquer pessoa pode pegar a COVID-19 e ficar gravemente doente, tendo a morte por consequência.

Conforme estudos de Carda et al. (2020), a COVID-19 possui diferentes manifestações clínicas, divididas em graus e podendo ser observado com maior frequência as seguintes manifestações: grau leve - sem dispneia, sem baixa saturação de oxigênio no sangue ( $\mathrm{SatO} 2$ ), com presença ou não de picos febril, perda de olfato e paladar; grau moderado - dispneia a pequenos

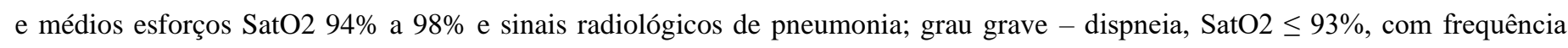
respiratória (FR)> 30 / min, progressão radiológica das lesões, necessidade de suplementação de $\mathrm{O} 2$, eventualmente com ventilação não invasiva; grau crítico - os pacientes necessitam de ventilação mecânica.

Em relação à construção e aplicação dos exercícios físicos a recomendação é que estes sejam de intensidade e volumes moderados (Jiménez-Pavón et al, 2020). Segundo as diretrizes do Colégio Americano de Medicina do Esporte (ACSM), onde traz que ao realizar exercícios físico regulares de intensidade moderada a vigorosa, irão melhorar as respostas imunológicas à infecção; diminuir a inflamação crônica de baixo grau, como também melhoras os marcadores imunológicos e inflamatório no COVID19 como também em várias outras doenças incluindo câncer, HIV, doenças cardiovasculares entre outras.

Nesse sentido, frente esses aspectos relacionados as doenças que podem vir a ter consequências mais graves mediantes ao COVID-19, é importante salientar a relevância da atividade física frente desse processo, onde segundo Alberti et al. (2021), a 
pratica de atividade física é de extrema valia, pois pode impedir o desenvolvimento de uma síndrome, conhecida como "Sara", que é síndrome da angústia respiratória aguda, tendo como características alguns sintomas, tais como, falta de ar, tosse, fraqueza muscular, e respiração rápida, sendo um dos fatores que abrange as complicações do COVID-19, haja vista, que a pratica de atividade física proporciona uma elevação da produção de enzimas superóxido dismutase, que tem associação a proteção do sistema cardiorrespiratório.

É importante ressaltar que toda construção e aplicação de exercícios físicos devem ser acompanhados por um Profissional de Educação Física, para que o mesmo possa mensurar carga e volume de treinamento, assim fazendo com que o sujeito tenha um treino seguro e significativo alcançando os objetivos desejados sejam eles estéticos ou imunológicos. O treinamento realizado de forma controlada e periodizada tem demonstrado melhorar a função imune (Jiménez-Pavón et al, 2020). Portanto, a prescrição de treinos de forma controlada e com constância tende a proporcionar melhora na função autoimune, promovendo respostas rápidas e eficazes contra as doenças, deixando o organismo mais preparado contra a COVID-19 (Lima, 2020).

$\mathrm{Na}$ ausência de variedade de equipamentos para os exercícios de força, o ACSM indica o uso de exercícios com peso corporal, uma opção que parece mais adequada para a aplicação de exercícios resistidos em casa, tendo em vista a recomendação de isolamento social devido à pandemia de COVID-19. Com isso, é importante salientar que todo exercício deve ser orientado por um profissional de Educação Física. Assim, o exercício físico moderado e regular deve ser especialmente praticado como medida de prevenção e recuperação de doenças A OMS (2020), recomenda que indivíduos pratiquem exercícios físicos de forma moderada, no mínimo, 150 minutos por semana. Haja vista que o exercício físico potencialmente melhora a saúde, o bem-estar, a aptidão física, o humor, a função cerebral, os sinais/sintoma, a efetividade do tratamento medicamentoso, entre outros.

Quadro 1: Programa de treinamento resistido calistênico baseado nas recomendações do ACSM (2018).

\begin{tabular}{|c|c|c|c|}
\hline SEGMENTO CORPORAL & EXERCÍCIO & VARIAÇÃO DE REGRESSÃO & $\begin{array}{l}\text { VARIAÇÃO DE } \\
\text { PROGRESSÃO }\end{array}$ \\
\hline \multirow{3}{*}{ Tronco inferior (core) } & Prancha Ventral & $\begin{array}{l}\text { Prancha Ventral (apoio dos } \\
\text { joelhos) }\end{array}$ & $\begin{array}{c}\text { Prancha Ventral (elevação dos } \\
\text { braços) }\end{array}$ \\
\hline & Prancha Lateral & $\begin{array}{c}\text { Prancha Lateral (apoio dos } \\
\text { joelhos) }\end{array}$ & $\begin{array}{l}\text { Prancha Lateral (abdução de } \\
\text { quadril) }\end{array}$ \\
\hline & Perdigueiro & Perdigueiro Unilateral & Perdigueiro dinâmico \\
\hline \multirow{3}{*}{$\begin{array}{l}\text { Tronco superior e membros } \\
\text { superiores }\end{array}$} & Flexão de braços & Flexão de braços com joelho & Flexão de braços tocando o chão \\
\hline & Remada suspensa & Remada com toalha (em pilastra) & Remada unilateral em suspensão \\
\hline & Flexão de ombros & Flexão de ombro aberta & Flexão de ombro fechada \\
\hline \multirow{3}{*}{ Membros inferiores } & $\begin{array}{c}\text { Agachamento } \\
\text { Afundo }\end{array}$ & $\begin{array}{l}\text { Agachamento com apoio } \\
\text { Afundo com apoio }\end{array}$ & $\begin{array}{l}\text { Agachamento com salto } \\
\text { Afundo deslocando }\end{array}$ \\
\hline & Bom dia (mãos na cintura) & Bom dia & Bom dia (mãos na nuca) \\
\hline & Tríceps sural unilateral & Tríceps sural bilateral & Tríceps sural com salto \\
\hline
\end{tabular}

Fonte: ACSM (2018). Elaborado pelos autores.

Neste momento de isolamento as atividades físicas realizadas em casa recomendam-se exercícios de fortalecimento muscular, alongamentos, exercícios de equilíbrio, com auxílio de procedimentos tecnológicos, tais como vídeos, aplicativos sempre com a supervisão e orientação do Profissional de Educação Física (Pitanga et al., 2020). Não esquecendo que as prescrições online 
devem contemplar todos os elementos importantes a uma prescrição e orientação para um programa de exercício físico, compreendendo ainda que o profissional mais bem colocado no ramo da Educação Física será aquele que se adapte melhor às necessidades e ao contexto vivido pela sociedade e que esteja sempre em busca de novos conhecimentos para seu melhor desempenho como profissional (Silva, 2020).

\section{Resultados e Discussão}

$\mathrm{Na}$ análise do nosso estudo, recomendamos atenção ao desenvolvimento de exercício e a prática excessiva do mesmo relacionado à qualidade de vida, tendo em vista que todo exercício físico deve ser orientado e supervisionado por um profissional de Educação Física, pois são atividades com volume e intensidade de forma sistematizada e adequada para o sujeito. Assim mostrando a partir de estudos recentes que o exercício é uma intervenção de baixo custo, que pode promover saúde em vários aspectos quando conscientemente realizado.

Quadro 2: Eixos temáticos e suas respectivas referências.

\begin{tabular}{|l|l|}
\hline EIXOS TEMÁTICOS & REFERÊNCIAS \\
\hline ATIVIDADE FÍSICA & $\begin{array}{l}\text { Mattos et al., 2020; Elesbão et al., 2020; Brasil, 2021; Pitanga, Beck, Pitanga, 2020; } \\
\text { Meneguci et al., 2016; Florêncio Júnior et al., 2020; OMS, 2020; Hall et al., 2020; } \\
\text { Alberti et al., 2021. }\end{array}$ \\
\hline EXERCÍCIO FÍSICO & $\begin{array}{l}\text { Carvalho et al., 2021; Mattos et al., 2020; Elesbão et al., 2020; Zawadzki, Stiegler e } \\
\text { Brasilino, 2019; Florêncio Júnior et al., 2020; Carvalho, 2021; ACMS, 2018; } \\
\text { Jiménez-pavón et al., 2020; Lima, 2020; Pitanga et al., 2020. }\end{array}$ \\
\hline COVID-19 & $\begin{array}{l}\text { OMS, 2020; Cortez et al., 2020; ECDC, 2020; Ladu et al., 2020; Alecrim, 2020; } \\
\text { Chen et al., 2020; Carda et al., 2020. }\end{array}$ \\
\hline CALISTENIA & Santos et al., 2020; ACSM, 2020. \\
\hline
\end{tabular}

Fonte: Elaborado pelos autores (2021).

Nesse contexto, é importante salientar que o quadro apresentado anteriormente, trata dos pontos chaves para o desenvolvimento do artigo, sendo dividido em quatro pontos principais, onde o primeiro e segundo são pontos que trabalham fatores bem específicos e semelhantes, nessa perspectiva trata-se da atividade física e o exercício físico, sendo assim, dois dos quatros pontos de suma relevância para desenvolvimento do trabalho. Assim, é importante frisar a importância da atividade física e exercício físico frente esse processo de pandemia da Covid-19, em especifico abordando olhar para saúde e qualidade de vida, sendo que, para Souza Filho e Tritany (2020) salientam que a atividade física, traz inúmeros benefícios, sendo esses, em curto e longo prazo, relacionados a diminuição de efeitos nocivos frente aos períodos de imobilidade muito relacionados ao contexto do COVID-19, além de fatores como doenças crônicas e suas comorbidades, e sua parte fundamental na melhoria no desfecho de quadro clinico mediante ao COVID-19, na saúde dos indivíduos no contexto social.

Assim, é possível ver o quanto é importante a relevância do exercício físico, no nosso contexto social, tão necessário de cuidados com a saúde e qualidade de vida, em um momento delicado que é esse da pandemia, sendo importante entender mediante ao que já foi expresso anteriormente, o papel fundamental que o exercício físico possibilita na vida dos indivíduos na sociedade. Mediante a isto, Pires et al. (2019) abordam que o exercício físico tem grande valia frente ao sistema imunológico, sendo desenvolvido de forma moderada a intensa, tendo uma resposta importante na parte imunológica, e diminuição das inflamações crônicas e diversos outros fatores relacionados a outras doenças, tais como, HIV, câncer, diabetes, obesidade e outras. Para corroborar com essa relação, Nogueira et al. (2020), frisam que sessões de atividades físicas é de fundamental valia frente a 
pessoas com infecções virais, pois o movimenta-se de maneira orientada pode melhorar as funções imunológicas, podendo prevenir ou atenuar malefícios maiores frente as infecções.

Dentro desse viés, o terceiro ponto de discussão no desenvolvimento do artigo, é sobre o COVID-19, que é um dos pontos centrais do trabalho, onde buscou analisar as relações da atividade física e exercício físico frente ao covid-19, que vem assolando mundialmente com diversas famílias, e é notável que um dos fatores que possibilite o melhoramento da saúde e qualidade de vida, seja antes ou pós covid-19, é a pratica de atividade física e exercício físico pelos indivíduos no contexto social. Nesse contexto, Nogueira et al. (2020) expressam que no contexto social estamos nos deparando com dois tipos de pandemia, a relacionada a inatividade física/sedentarismo e o COVID-19, onde a inatividade física vem em um longo percurso, tendo seus malefícios aumentados frente ao COVID-19, que faz as pessoas se movimentarem cada vez menos, sendo que é necessário que a prática de atividade física e exercício físico, seja desenvolvidas para melhoramento desses pontos maléficos que se deparamos no contexto social.

A calistenia é um ponto primordial para a prática de exercícios físicos em casa, pois o mesmo não necessita de aparelhos ou pesos para ser trabalhada, é um exercício que utiliza somente o peso corporal do sujeito, segundo Machado (2017) a Calistenia é a prática de exercícios somente com o peso corporal, movimentos ritmados e uma elaboração da sessão de treino obedecendo a uma regra metodológica. Diante disso é de total importância a presença de um profissional mesmo online de Educação Física para orientar e montar um treino adequado ao sujeito, respeitando, mensurando o ritmo do treino, prevenindo possíveis lesões e exageros, para que assim o sujeito tenha o melhor desempenho e benefícios no treino mesmo estando em casa. Assim, para corroborar com o que foi exposto, La Escala Teixeira (2019) frisa que os exercícios calistênicos são desenvolvidos com próprio peso corporal, abordando padrões de movimentos que podem ser de forma cíclica ou não cíclica, sendo com carga alta ou baixa, sendo que, pode ser realizada por qualquer público, seja iniciante ou atletas de alta desempenho, além do baixo custo e praticidade.

\section{Considerações Finais}

Percebe-se, por meio deste estudo, que a prática de atividade física e exercícios físicos é fundamental para a qualidade de vida da população em geral, contendo inúmeros benefícios que proporciona principalmente no controle de doenças crônicas, como a Hipertensão e Diabetes. Uma vida saudável requer atitudes com hábitos saudáveis, principalmente atividade físicas regulares, que sem dúvida proporcionará melhor qualidade de vida e maior longevidade. Entende-se que os hábitos de vida adquiridos pela sociedade moderna, criou um ambiente propício para hábitos sedentários, cabe agora aos profissionais da área de saúde modificar está situação que se apresenta de forma preocupante, o profissional de Educação Física deve sempre buscar meios de proporcionar a sociedade e seus clientes hábitos saudáveis, produzindo assim uma visão e uma mudança aos hábitos de vida e consequentemente então uma melhor qualidade de vida do sujeito na sociedade moderna.

Diante da pandemia do COVID-19 o comportamento sedentário se tornou muito mais evidente devido ao isolamento social, acarretando em possíveis problemas de saúde devido a inatividade física e a falta de práticas de exercícios regulares semanais ou diariamente, onde a partir daí algumas pessoas optaram por fazer calistenia, assim o profissional de Educação Física teve de se reinventar com as novas tecnologias e dar aulas e orientações online para seus clientes, para que mesmos isolados as pessoas possas fazer exercícios diariamente e assim manter uma vida mais ativa e saudável mesmo estando dentro de casa. É notório que as informações discutidas no artigo podem ainda ser utilizadas em trabalhos futuros e esclarecimento de como foi a relação e vivencia das pessoas que enfrentaram o isolamento social devido ao COVID-19, mostrando que podemos continuar ativos mesmo estando dentro de casa. 
Portanto, conclui-se que mediante aos autores pesquisados, a relevância que atividade física e o exercício físico diante de uma pandemia, visando os aspectos da saúde e qualidade de vida, pois possibilita inúmeros benefícios, além de ser uma ferramenta prática podendo ser desenvolvida dentro de seu âmbito familiar, sendo de baixo custo e de grande resultados, diante de uma orientação e acompanhamento de um profissional de Educação Física, enfim, trouxe inúmeros fatores que proporciona aos cidadãos no contexto social, que necessitam urgentemente de mais possibilidades e cuidados com a saúde e consequentemente possam adquirir uma boa qualidade de vida.

\section{Referências}

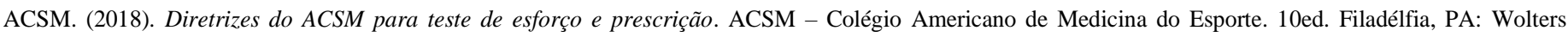
Kluwer.

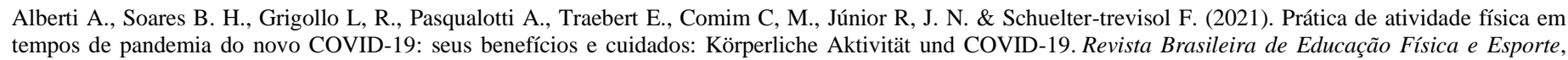
35(2), 375-380.

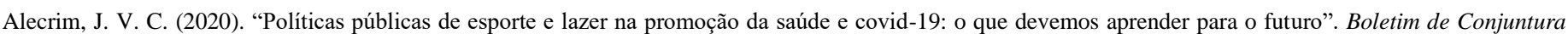
(BOCA), 2(5).

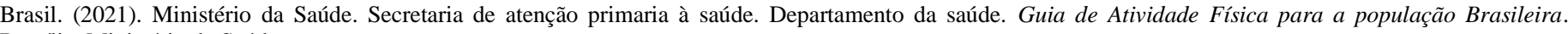
Brasília: Ministério da Saúde.

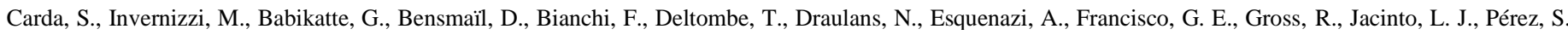

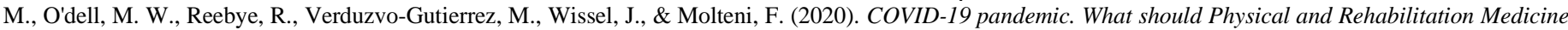

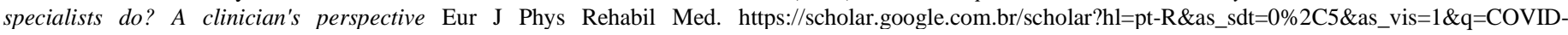
$19+$ pandemic. + What+should+Physical+and+Rehabilitation+Medicine+specialists+do\%3F+A+clinician $\% 27 \mathrm{~s}+$ perspective $\& b t n G=\# d=g s \_q a b s \& u=\%$ 23p\%3D7bRkBOxY4nUJ.

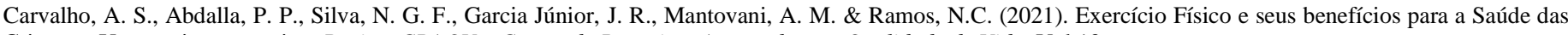
Crianças: Uma revisão narrativa. Revista CPAQV - Centro de Pesquisas Avançadas em Qualidade de Vida. Vol.13.

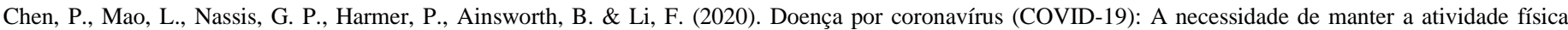
regular tomando precauções. J Sport Heal Sci https://doi.org/10.1016/j.jshs.2020.02.001.

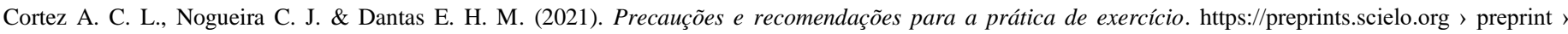
download.

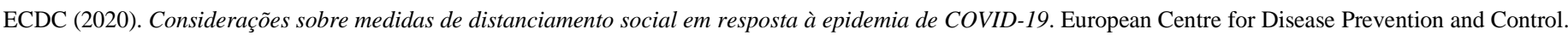
https://www.ecdc.europa.

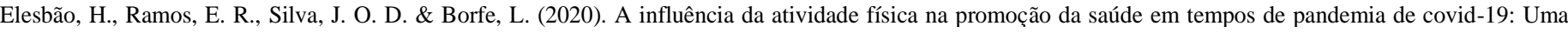
revisão narrativa. Revista Interdisciplinar de Promoção da Saúde, 3(4),

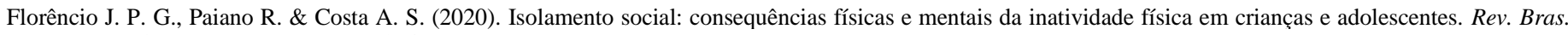
Ativ. Fis. Saúde.; 25:e0115. 10.12820/rbafs.25e0115.

GIL, A. C. (2010). Como elaborar projeto de pesquisa. (5a ed.), Atlas.

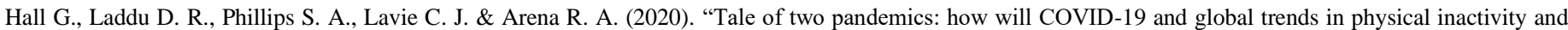
sedentary behavior affect one another?". Progress Cardiovascular Diseases, 63.

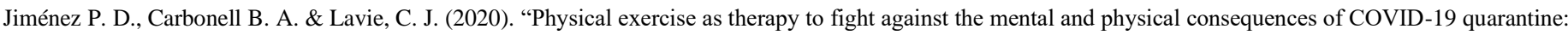
Special focus in older people published online a head of print". Journal of Physical Activity and Health, 63(3).

LA ESCALA T. C.V. (2019). Hiit calistênico: Treinamento intervalado de alta intensidade com o peso do corpo. Edição do Autor.

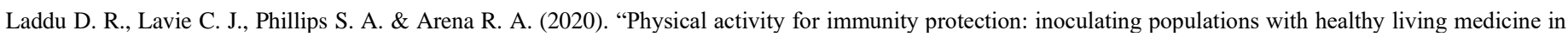
preparation for the next pandemic". Progress Cardiovascular Diseases, 63(2).

Lima, L. C. J. (2020) Alimentação saudável e exercícios físicos em meio a pandemia da COVID-19. Boletim de Conjuntura (BOCA) ano II, 3(9).

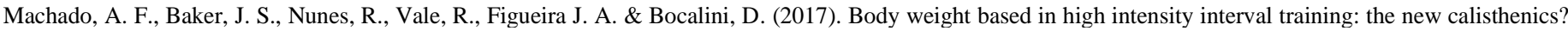
MTP \& Rehab journal. 15:448. 10.17784/mtprehabjournal.2017.15.448. 
Samuel M. M., Déborah S. P., Thereza M. M. M., Virna R. F. C. \& Ricardo H. G. (2020). Recomendações de atividade física e exercício físico durante a pandemia Covid-19: revisão de escopo sobre publicações no Brasil. Revista Brasileira de Atividade Física \& Saúde, 25, 1-12. https://doi.org/10.12820/rbafs.25e0176.

Meneguci, J., Garcia, C. A., Sasaki, J. E. \& Júnior, J. S. V. (2016). Atividade física e comportamento sedentário: fatores comportamentais associados à saúde de idosos. Arquivos de Ciências do Esporte, 4(1):27-28.

Nahas M. V. (2010). Atividade física, saúde e qualidade de vida: conceitos e sugestões para um estilo de vida ativo. (5a ed.), Paraná.

Nogueira, C. J., Antônio C. L. C., Silvânia M. O. L. \& Estélio H. M. D. (2020). Precauções e recomendações para a prática de exercício físico em face do COVID19: uma revisão integrativa.

OMS. (2021). Folha informativa sobre COVID-19. https://www.paho.org/pt/covid19.

OMS. (2020). Organização Mundial da Saúde. Diretrizes da OMS para Atividade Física e Comportamento Sedentário: num piscar de olhos. https://apps.who.int/iris/bitstream/handle/10665/337001/9789240014886-

por.pdf?sequence=102\&isAllowed=y\#: :text=Para\%20sa \% 3\%BAde\%20e\%20bem\%2Destar,dia\%20para\%20crian\%C3\%A7as\%20e\%20adolescentes

Pires E. A. G., Duarte M. F. S., Pires M. C. \& Souza G. S. (2019). Hábitos de atividade física e o estresse em adolescentes de Florianópolis-SC, Brasil. revista brasileira de cine antropometria e desenvolvimento humano, 12(1).

Pitanga F. J.G., Beck C. C. \& Pitanga C. P.S. (2020). "Should physical activity be considered essential activity during the covid-19 pandemic?" International Journal of Cardiovascular Sciences, 33(4).

Pitanga F. J.G., Beck C. C. \& Pitanga C. P.S. (2020). Atividade física e redução do comportamento sedentário durante a pandemia do Coronavírus. Arq Bras Cardiol. published online ahead of Print.

Richardson, R. J. (2011). Pesquisa social: métodos e técnicas. Atlas.

Santos A. A. P., Gomes W. N. P., Santos R. S. \& Landim L. A. S. R. (2020). Calistenia: uma revisão. Research, Society and Development, 9(10), e9679109371e9679109371.

Silva A. L. L. (2020). Prescrição do Exercício Físico em tempos de Pandemia. Revista Brasileira do Esporte Coletivo - a. 4. 4(2).

Souza F., Breno A. B., \& Tritany, É. F. (2020). COVID-19: importância das novas tecnologias para a prática de atividades físicas como estratégia de saúde pública. Cadernos de Saúde Pública, 36, e00054420.

Zawadzki, D., Stiegler, N. F. F. \& Brasilino, F. F. (2019). Aptidão e a atividade física relacionados à saúde de adolescentes entre 11 a 14 anos. Revista Brasileira de Prescrição e Fisiologia do Exercício, 13(83), 444-453. 